\title{
Monitoring $\alpha 4 \beta 7$ integrin expression on circulating CD4 + T cells as a surrogate marker for tracking intestinal CD4 + T-cell loss in SIV infection
}

\author{
X Wang ${ }^{1}, \mathrm{H} \mathrm{Xu}^{1}$, AF Gill ${ }^{1}$, B Pahar ${ }^{1}$, D Kempf ${ }^{1}$, T Rasmussen ${ }^{1}$, AA Lackner ${ }^{1}$ and RS Veazey ${ }^{1}$
}

Intestinal CD4 + T cells are rapidly and profoundly depleted in human immunodeficiency virus (HIV)-infected patients and simian immunodeficiency virus (SIV)-infected macaques. However, monitoring intestinal cells in humans is difficult, and identifying surrogate markers in the blood, which correlate with loss or restoration of intestinal CD4 + T cells could be helpful in monitoring the success of therapeutic strategies and vaccine candidates. Recent studies indicate HIV utilizes the intestinal homing molecule $\alpha 4 \beta 7$ for attachment and signaling of CD4 + T cells, suggesting this molecule may have a central role in HIV pathogenesis. Here, we compared $\beta 7^{\mathrm{HIGH}}$ integrin expression on CD4 + T cells in blood with loss of CD4 + T cells in the intestine of macaques throughout SIV infection. The loss of $\beta 7^{\mathrm{HIGH}} \mathrm{CD} 4+\mathrm{T}$ cells in blood closely paralleled the loss of intestinal CD4 + T cells, and proved to be a more reliable marker of intestinal CD4 + T-cell loss than monitoring CCR5 + memory CD4 + T cells. These data are consistent with a recent hypothesis that $\alpha 4 \beta 7$ has a role in the selective depletion of intestinal CD4 + T cells, and indicate that monitoring $\beta 7^{\mathrm{HIGH}}$ expression on CD4 + T cells in the blood may be a useful surrogate for estimating intestinal CD4 + T cell loss and restoration in HIV-infected patients.

\section{INTRODUCTION}

The intestinal tract is known to be a major site of early human immunodeficiency virus (HIV) infection and replication, and is likely a major reservoir for viral persistence, particularly in patients on anti-retroviral therapy. ${ }^{1,2}$ Thus, a number of investigators are sampling intestinal CD $4+\mathrm{T}$ cells to monitor response to anti-retroviral therapy. ${ }^{1,3,4}$ Furthermore, understanding the dynamics of HIV infection and replication, specifically in mucosal tissues, is fundamental to understanding the pathogenesis of HIV infection, and probably to the development of an effective HIV-1 vaccine. ${ }^{5}$ For these and other reasons, there is an emerging consensus that measuring intestinal mucosal immune responses may be important for measuring the effectiveness of HIV-1 vaccines and treatment strategies.

However, examining intestinal tissues in humans is problematic, and not without risk. Intestinal biopsies require specialized equipment and skills, and it is unlikely that they can be routinely performed in the field, especially in the context of a large vaccine trial. Therefore, it would be very useful to identify a surrogate marker for tracking changes in mucosal CD $4+\mathrm{T}$ cells which could be routinely measured in blood to predict responses to therapy, vaccines, or progression to AIDS.

The integrin $\alpha 4 \beta 7$ mediates lymphocyte migration to the intestine through interaction with the mucosal addressin cell adhesion molecule-1 (MAdCAM-1), which is predominantly expressed on venules in the gut-associated lymphoid tissue (GALT) and intestinal lamina propria. ${ }^{6,7}$ Thus, cells expressing $\alpha 4 \beta 7$ in the blood are believed to traffic predominantly to the intestine. Recently, HIV-1 gp120 was shown to bind and signal through $\alpha 4 \beta 7$, which provides an additional explanation for HIV's selective tropism for the intestinal immune system, in addition to the preponderance of CCR $5+$ memory phenotype CD4 + T cells in this location. ${ }^{8-10}$ Furthermore, HIV-1 binding to $\alpha 4 \beta 7$ triggers cellular activation and may facilitate selective infection of these cells, or the formation of viral synapses, which may facilitate cell-to-cell HIV-1 transmission. ${ }^{5}$ We thus hypothesized that the level of circulating $\alpha 4 \beta 7+\mathrm{CD} 4+\mathrm{T}$ cells in blood would correlate with the level of CD $4+\mathrm{T}$ cells remaining in the intestine, and may serve as a surrogate marker for monitoring intestinal CD4 + T-cell depletion or reconstitution in simian

${ }_{1}^{1}$ Tulane National Primate Research Center, Tulane University School of Medicine, Covington, Louisiana, USA. Correspondence: R Veazey, (rveazey@tulane.edu)

Received 10 June 2009; accepted 24 June 2009; published online 26 August 2009. doi:10.1038/mi.2009.104 
immunodeficiency virus (SIV)-infected macaques, and by inference, HIV-infected patients.

Unfortunately, anti-human/non-human primate monoclonal antibodies to $\alpha 4 \beta 7$ are limited, and not commercially available. However, cross-reactive antibodies to the integrin $\beta 7$ are available, and although this detects both $\alpha \mathrm{E} \beta 7$ (intestinal epithelial) as well as $\alpha 4 \beta 7$ (lamina propria) lymphocytes, $\alpha \mathrm{E} \beta 7$ is rarely expressed in the blood, and its role in homing is less clear. ${ }^{11,12}$ We thus used $\beta 7$ integrin alone, and in combination with CD49d ( $\alpha 4$ integrin) to examine and compare mucosal homing lymphocytes.

\section{RESULTS}

\section{Distribution of $\beta 7^{\mathrm{HIGH}} \mathrm{CD} 4+\mathrm{T}$ cells in tissues of normal rhesus macaques}

To confirm that $\beta 7^{\mathrm{HIGH}}$ integrin expression was specific for intestinal tissues and cells homing to gut in macaques, the expression and distribution of $\beta 7^{\mathrm{HIGH}} \mathrm{CD} 4+\mathrm{T}$ cells was compared in multiple regions of gut, lymph nodes, spleen, thymus, bone marrow, and peripheral blood of normal rhesus macaques. The majority of $\beta 7^{\mathrm{HIGH}} \mathrm{CD} 4+\mathrm{T}$ cells were intestinal lamina propria lymphocytes in the jejunum, ileum, and colon as well as intestinal intraepithelial lymphocytes. Substantial numbers of $\beta 7{ }^{\mathrm{HIGH}} \mathrm{CD} 4+\mathrm{T}$ cells were also detected in the blood and mesenteric lymph nodes. In contrast, very few (0.7 to $1.5 \%)$ $\beta 7^{\mathrm{HIGH}} \mathrm{CD} 4+\mathrm{T}$ cells were detected in the spleen, and $\beta 7^{\mathrm{HIGH}}$ expression was practically absent in the thymus and bone marrow (Figure 1). Interestingly, only a fraction ( 15\%) of intestinal $\mathrm{CD} 4+$ cells were $\beta 7^{\mathrm{HIGH}}$ suggesting that this integrin may be downregulated after the cells reach their target tissues, which has been shown for murine CD8 + T cells. ${ }^{13,14}$ However, the majority of intestinal cells expressed low or intermediate levels of $\beta 7$ (not shown). Importantly, few $\beta 7^{\mathrm{HIGH}} \mathrm{CD} 4$ cells were present in the axillary's LN, despite large numbers being detected in the

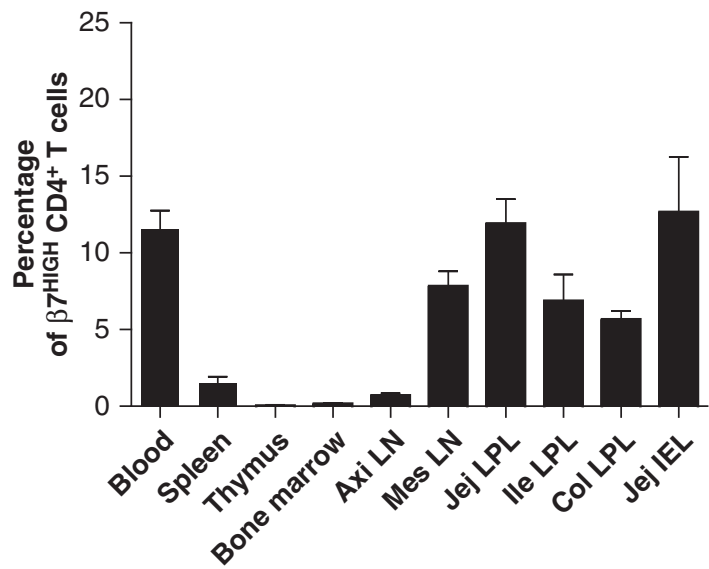

Figure 1 Distribution of $\beta 7^{\mathrm{HIGH}}$ integrin expression on CD4 + T cells from various tissues in normal (non-infected) rhesus macaques. Note that the vast majority of $\beta 7^{\mathrm{HIGH}} \mathrm{CD} 4+\mathrm{T}$ cells reside in intestine tissues (IEL and LPL) and in blood. Also note that far more $\beta 7^{\mathrm{HIGH}} \mathrm{CD} 4+\mathrm{T}$ cells are present in the mesenteric lymph node compared with axillary lymph nodes, thymus, or bone marrow. Data represent means \pm s.e.m. from five normal macaques examined. IEL, intestinal intraepithelial lymphocytes; LPL, lamina propria lymphocytes. mesenteric LN (Figure 1). Combined, this validated that $\beta 7^{\mathrm{HIGH}}$ expression in macaques was primarily limited to intestines and tissues involved in the gut-homing pathway (mesenteric lymph nodes and blood).

\section{CD4 + T cells expressing $\beta 7^{\mathrm{HIGH}}$ have a "memory" phenotype}

Both HIV-1 and SIV selectively infect and deplete memory CD4 + T cells in early infection. ${ }^{8,15-18}$ Thus to determine if $\alpha 4 \beta 7^{\mathrm{HIGH}} \mathrm{CD} 4+\mathrm{T}$ cells express a memory phenotype, we examined the coexpression of CD45RA (a naive cell marker) CD95 (a memory marker) and CD49d ( $\alpha 4$ integrin) on CD4+ $\beta 7^{\mathrm{HIGH}} \mathrm{T}$ cells. In all tissues, $\beta 7^{\mathrm{HIGH}} \mathrm{CD} 4+\mathrm{T}$ cells were essentially all memory cells (CD45RA- and CD95 +)(Figure 2). In contrast, $\beta 7$ "intermediate" $\mathrm{CD} 4+\mathrm{T}$ cells were all naive cells $(\mathrm{CD} 45 \mathrm{RA}+, \mathrm{CD} 95-)$. In the blood, all $+\beta 7^{\mathrm{HIGH}} \mathrm{CD} 4+$ cells also coexpressed CD28, consistent with a "central memory" phenotype (data not shown). Furthermore, essentially all $\beta 7^{\mathrm{HIGH}}$ $\mathrm{CD} 4+\mathrm{T}$ cells in the blood coexpressed the $\alpha 4$ integrin (Figure 2). Combined, these results indicated that circulating $\beta 7^{\mathrm{HIGH}} \mathrm{CD} 4+\mathrm{T}$ cells in blood and lymph nodes were all $\alpha 4 \beta 7+$ central memory cells, and likely homing to intestinal tissues. Thus, we hypothesized that tracking this cell subset in the blood could predict changes in $\mathrm{CD} 4+\mathrm{T}$ cells in the intestinal tract.

\section{Loss of circulating $\beta 7^{\mathrm{HIGH}}$ CD4 $+\mathrm{T}$ cells parallels the loss of intestinal CD4 + T cells in SIV infection}

To determine if changes in $\beta 7^{\mathrm{HIGH}} \mathrm{CD} 4+\mathrm{T}$ cells reflected changes in intestinal CD4 + T cells, we compared cells from the blood and intestine of animals at various stages of SIV infection. As expected, intestinal CD $4+\mathrm{T}$ cells were markedly and persistently depleted within 13 days after infection in all animals examined (Figure 3). Although a mean of $38 \%$ of lymphocytes in the intestine were CD $4+$ before infection, only $5-15 \%(9 \pm 3.2)$ of the remaining lymphocytes were $\mathrm{CD} 4+$ by 13 days of infection (Figure 3). Furthermore, intestinal CD $4+\mathrm{T}$ cells remained depleted in virtually all animals infected for more than 13 days, regardless of the stage of infection (Figure 3). In addition, $\beta 7^{\mathrm{HIGH}} \mathrm{CD} 4+\mathrm{T}$ cells were also rapidly and selectively depleted in the intestine in acute SIV infection (Figures 3 and 4). Note that although abundant $\mathrm{CD} 4+$ and $\beta 7+$ cells are detected in the intestine of normal macaques (Figure 3a) most of both phenotypes are selectively eliminated within days of infection (Figure $3 \mathbf{b}$ ). Finally, in animals infected with SIV or SHIV that were controlling plasma viremia, $\beta 7{ }^{\mathrm{HIGH}} \mathrm{CD} 4+\mathrm{T}$ cells in the intestine were only partially restored (Figure $3 \mathbf{b}$ ). Notably, $\beta 7^{\mathrm{HIGH}} \mathrm{CD} 8+$ $\mathrm{T}$ cells were not depleted in the intestine or any other tissues at any stage of SIV infection (Figure 3 and data not shown). Furthermore, immunohistochemistry of intestinal tissues from animals in early SIV infection suggested a dramatic loss of $\alpha 4 \beta 7+\mathrm{CD} 4+\mathrm{T}$ cells (but not CD $8+\mathrm{T}$ cells) consistent with selective lysis of these cells in the lamina propria (Figure 4).

As $\alpha 4 \beta 7$ directs homing of $T$ cells specifically to intestinal tissues, and likely has a major role in the maintenance and/or 
a
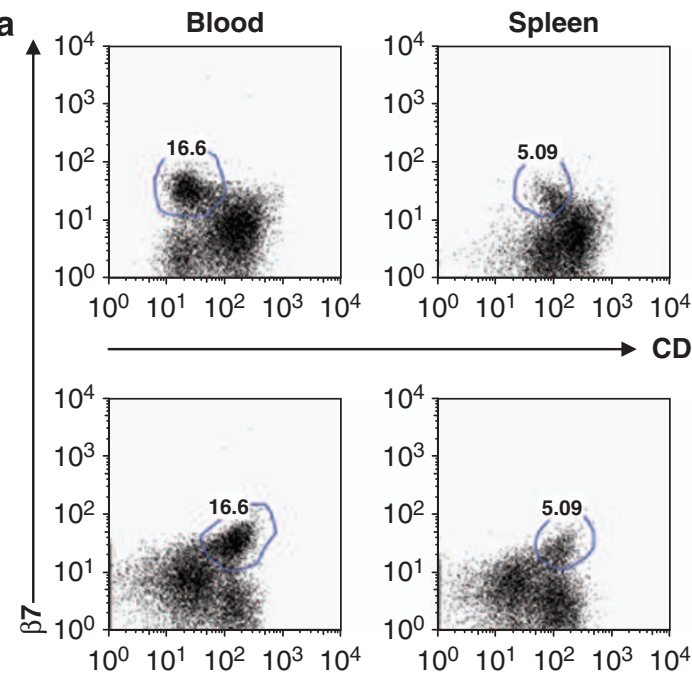
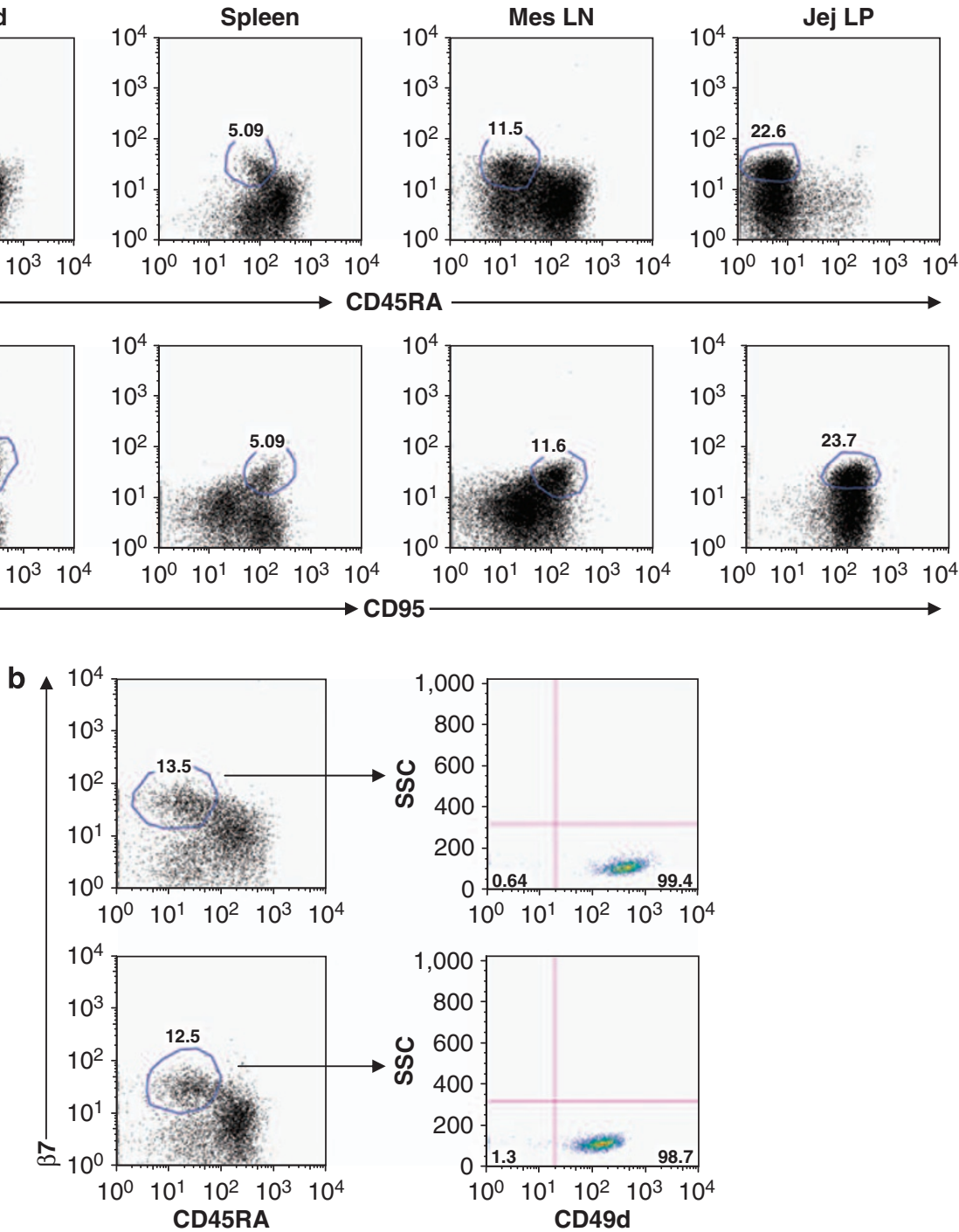

Figure 2 Phenotyping $\beta 7^{\mathrm{HIGH}} \mathrm{CD} 4+\mathrm{T}$ cells in rhesus macaques. (a) Coexpression of naive (CD45RA) and memory (CD95) markers with $\beta 7$ HIGH integrin expression on CD4 + T cells from various tissues of representative macaques. Note that although the majority of lymphocytes express low or intermediate levels of $\beta 7$, essentially all $\beta 7^{\mathrm{HIGH}} \mathrm{CD} 4+\mathrm{T}$ cells (delineated by oval regions) have a memory (CD45RAnegCD95 +) phenotype. (b) Gating through $\beta 7^{\mathrm{HIGH}} \mathrm{CD} 4+\mathrm{T}$ cells in two representative animals shows that essentially all $\beta 7^{\mathrm{HIGH}} \mathrm{CD} 4+\mathrm{T}$ cells are also $\alpha 4^{\mathrm{HIGH}}\left(\mathrm{CD} 49 \mathrm{~d}^{\mathrm{HIGH}}\right)$, indicating that almost $\beta 7^{\mathrm{HIGH}} \mathrm{CD} 4+\mathrm{T}$ cells in the blood are $\alpha 4 \beta 7+$. All plots were generated by gating through lymphocytes, and then through CD4+ T cells.

reconstitution of intestinal CD4 $+\mathrm{T}$ cells, we hypothesized that a loss of circulating $\beta 7^{\mathrm{HIGH}} \mathrm{CD} 4+\mathrm{T}$ cells would correlate with the depletion and persistent loss of intestinal CD4 + T cells. As shown in Figure 5 detectable decrease in $\beta 7{ }^{\mathrm{HIGH}} \mathrm{CD} 4+\mathrm{T}$ cells was observed in blood by 8 days of infection, and levels were significantly $(P<0.01)$ lower than uninfected controls by 10 days of infection. Moreover, $\beta 7^{\mathrm{HIGH}} \mathrm{CD} 4+$ lymphocytes continued to decrease in blood in parallel with intestinal CD4 + $\mathrm{T}$ cells throughout infection. In fact, levels of $\beta 7^{\mathrm{HIGH}} \mathrm{CD} 4+$ $\mathrm{T}$ cells in blood closely reflected levels of intestinal CD4 $+\mathrm{T}$ cell changes throughout infection (Figure 5). Eventually, CD4 + T cells expressing $\beta 7^{\mathrm{HIGH}}$ in blood were essentially eliminated in macaques with AIDS, as were intestinal CD4 $+\mathrm{T}$ cells in these animals. Although there was a trend in chronic, asymptomatic infected animals to have higher levels of intestinal CD4 $+\mathrm{T}$ cells than macaques infected for 21 days (Figure 5), these differences were non-significant. However, in cohorts of animals controlling infection (with undetectable plasma viremia) $\beta 7^{\mathrm{HIGH}} \mathrm{CD} 4+$ $\mathrm{T}$ cells were partially restored, which also directly correlated with levels of intestinal CD4+ T-cell restoration (Figure 5). Combined, this suggests that circulating $\beta 7^{\mathrm{HIGH}}$ cells may have a role in restoring intestinal $\mathrm{CD} 4+\mathrm{T}$ cells, and tracking this $\mathrm{T}$-cell subset in blood may be a useful surrogate to directly measuring intestinal CD4 + T cells in HIV-1 infection.

\section{Comparison of the dynamics of $\beta 7^{\mathrm{HIGH}}$ and CCR5 + CD4 + $T$ cells in blood during acute SIV infection}

We and others previously hypothesized that the rapid and selective depletion of intestinal CD4 + T cells was due to a combination of their state of activation and expression of high levels of CCR5. ${ }^{8,15-17,19-21}$ Evidence for this included showing that activated CD $4+$ CCR5 + memory T cells were eliminated at a 


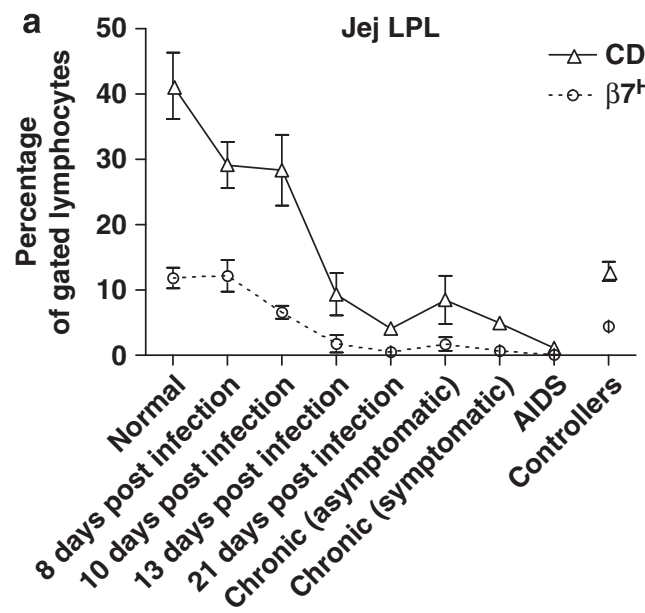

b
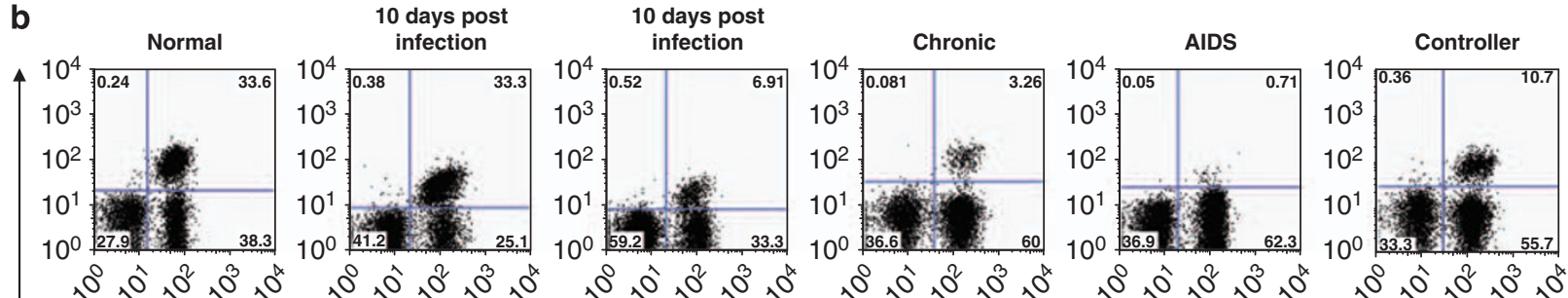

$10^{\circ}, 0^{1}, 0^{2}, 0^{3}, 0^{20}$

$10^{0}, 0^{x}, 0^{2}+0^{3}+0^{x}$

$10,0^{0}+0^{2}+0^{3}+0^{0}$

$10^{0}, 1^{2}+0^{2}+0^{3}, 0^{x}$
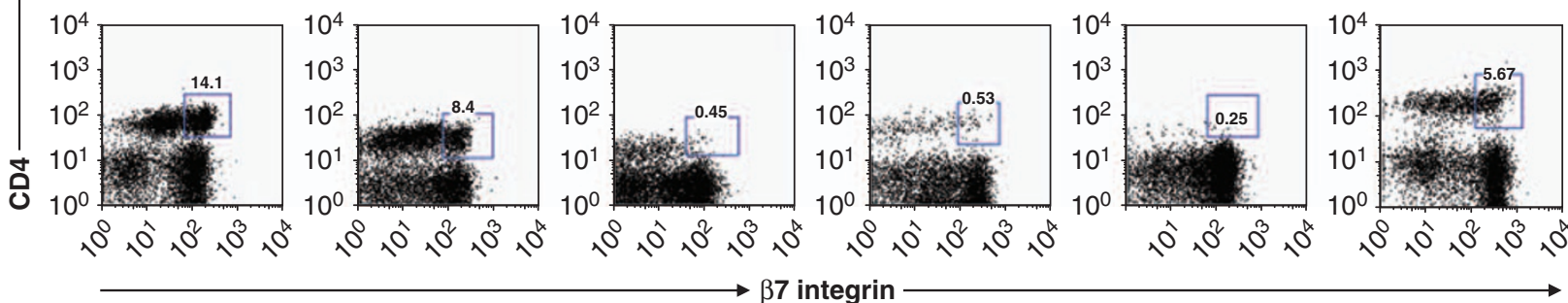

Figure 3 Changes in intestinal CD4 + CD3 + T cells and $\beta 7{ }^{\mathrm{HIGH}} \mathrm{CD} 4+\mathrm{T}$ cells throughout SIV infection. (a) Changes in intestinal CD4 + CD3 + T cells (black line) and $\beta 7^{\mathrm{HIGH} C D} 4+\mathrm{T}$ cells (dotted line) in SIV-infected macaques throughout infection. As expected, intestinal CD4+ T cells are markedly and persistently depleted by 13 days of inoculation, and this depletion persists throughout infection. $\beta 7^{\mathrm{HIGH}} \mathrm{CD} 4+\mathrm{T}$ cells are similarly persistently depleted in the intestine (dotted line), however, in animals controlling plasma viremia (controllers) there is a partial reconstitution of intestinal CD4+ $T$ cells and as $\beta 7^{\mathrm{HIGH}} \mathrm{CD} 4+\mathrm{T}$ cells. (b) Dot plots from a representative macaque showing a marked loss of CD4 + T cells (top panels) and a profound and selective loss of $\beta 7^{\mathrm{HIGH}} \mathrm{CD} 4+\mathrm{T}$ cells (bottom panels) in the intestine. Error bars in a indicate s.e.m. SIV, simian immunodeficiency virus.

higher rate than CCR5neg CD4 + T cells in the intestine. ${ }^{8,15,16}$ In addition, a selective loss of CD $4+$ CCR $5+\mathrm{T}$ cells in blood can be detected in individual macaques, at least in early SIV infection. ${ }^{8,15,16}$ However, as CCR 5 is expressed on activated memory $\mathrm{T}$ cells in most tissues, and not restricted to intestinal $\mathrm{T}$ cells, ${ }^{22}$ we hypothesized that tracking $\beta 7^{\mathrm{HIGH}} \mathrm{CD} 4+\mathrm{T}$ cells in the blood could be a better surrogate marker for monitoring intestinal $\mathrm{CD} 4+\mathrm{T}$-cell depletion. We thus compared the dynamics of circulating $\beta 7{ }^{\mathrm{HIGH}} \mathrm{CD} 4+\mathrm{T}$ cells with CCR $5+\mathrm{CD} 4+\mathrm{T}$ cells in the blood in normal and SIV-infected animals with changes in intestinal CD $4+\mathrm{T}$ cells. In normal macaques, percentages of circulating $\beta 7^{\mathrm{HIGH}} \mathrm{CD} 4+\mathrm{T}$ cells were much higher than CCR $5+$ CD $4+T$ cells (Figure 6a). Following SIV infection, both populations were rapidly and persistently depleted throughout infection, particularly in animals with AIDS. However, in animals controlling infection, blood CD $4+\mathrm{CCR} 5+\mathrm{T}$ cells had returned to levels similar to uninfected controls $(P>0.05)$, Thus,
CD4 + CCR $5+$ T cells in the blood were not reflective of the level of intestinal CD4 + T-cell depletion. In contrast, blood $\beta 7^{\mathrm{HIGH}} \mathrm{CD} 4+\mathrm{T}$ cells remained significantly lower in these "controllers" compared with uninfected animals $(P<0.01)$ and the level of blood $\beta 7^{\mathrm{HIGH}} \mathrm{CD} 4+\mathrm{T}$ cells closely correlated with levels of $\mathrm{CD} 4+\mathrm{T}$ cells remaining in the intestine. Finally, the rate and magnitude of $\beta 7^{\mathrm{HIGH}} \mathrm{CD} 4+\mathrm{T}$-cell depletion in the blood was much higher than in CCR $5+\mathrm{CD} 4+\mathrm{T}$ cells. As shown in Figure $\mathbf{6 b}$, the slope of decline was -0.8 for circulating $\beta{ }^{\mathrm{HIGH}} \mathrm{CD} 4+\mathrm{T}$ cells, vs. -0.3 for CCR $5+\mathrm{CD} 4+\mathrm{T}$ cells, indicating a more rapid decline of the former in early infection.

We also compared the dynamics of both circulating $\beta 7^{\mathrm{HIGH}} \mathrm{CD} 4+\mathrm{T}$ and $\mathrm{CCR} 5+\mathrm{CD} 4+\mathrm{T}$ cells to changes in intestinal $\mathrm{CD} 4+\mathrm{T}$ cells by linear regression analysis (Figure 7). Although both populations showed significant correlations with the intestinal CD $4+\mathrm{T}$ cell loss, the $r^{2}$ value of the $\beta 7{ }^{\mathrm{HIGH}} \mathrm{CD} 4+\mathrm{T}$ cells was 0.6 , whereas for CCR $5+\mathrm{CD} 4+\mathrm{T}$ 


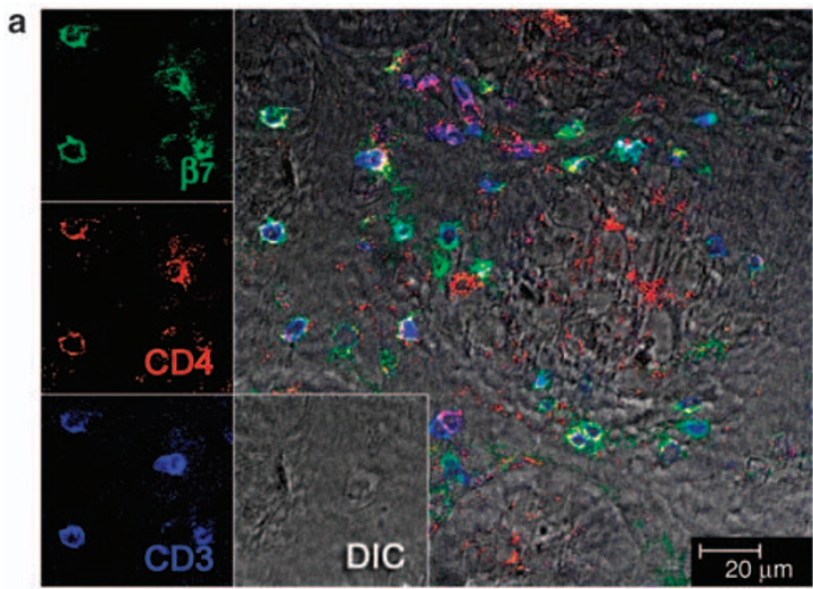

b

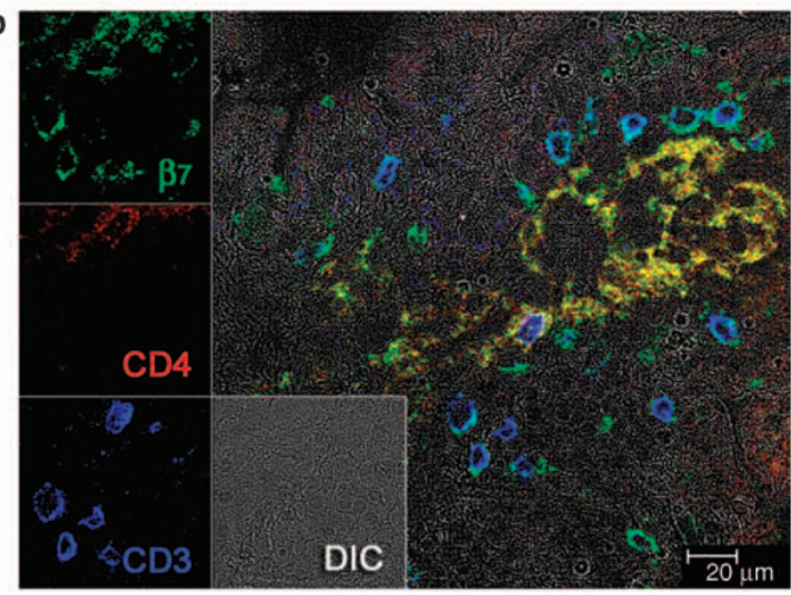

Figure 4 Visualization of $\beta 7+T$ cells in normal and SIV infected macaques. (a) Confocal microscopy of immunohistochemically stained jejunum sections showing $\beta 7$ (green), CD4 (red), and CD3 (blue) expression. Note that $\beta 7$ is normally expressed on T lymphocytes (CD3) in the intestinal mucosa. Also note that both $\beta 7+C D 4+C D 3+$ (yellowgreen/blue) $T$ cells and $\beta 7+C D 3 n e g$ cells (green-blue; presumably CD8 + T cells) are clearly evident in the intestine of normal macaques. (b) Jejunum section from an SIV-infected macaque 21 days after SIVmac251 inoculation. Note that both CD4 + (red) and CD4+ $\beta 7+$ (yellow-green/blue) T cells are now "missing" and yellow-green staining is limited to amorphous cellular debris, suggestive of marked, yet selective necrosis and lysis of CD3 $+C D 4+\beta 7+T$ cells. Note that abundant $C D 3+C D 4 n e g, \beta 7 \pm$ cells remain, indicating that non CD4+ T cells $(C D 8+)$ are spared. SIV, simian immunodeficiency virus.

cells it was 0.3 (Figure 7). Combined, this suggests that circulating $\beta{ }^{\mathrm{HIGH}} \mathrm{CD} 4+\mathrm{T}$ cells may be a better surrogate marker for monitoring intestinal CD4 + T-cell depletion than circulating CCR5 + CD $4+$ T cells.

\section{DISCUSSION}

It is well established that SIV and HIV-1 primarily target intestinal CD4 + T cells, particularly in early infection. ${ }^{2,17,20,23,24}$ However, the intestinal mucosal immune system consists of distinct immune inductive and effector compartments, both harboring large numbers of CD4 + T cells, but with different phenotypic and functional characteristics. Understanding the nature of these distinct sites and cells is particularly important for understanding the pathogenesis of HIV-1 infection.
Large numbers of "resting, naive" CD4 + T cells reside in intestinal immune inductive sites (Peyer's patches and organized lymphoid follicles) also known as GALT, which are distributed primarily in the terminal small intestine (ileum) and large intestine. When resting $\mathrm{T}$ cells in GALT become activated through exposures to antigens they rapidly leave the GALT, migrate through the mesenteric lymph nodes, briefly recirculate in the blood, and eventually "home" to the diffuse intestinal lamina propria, which is distributed throughout the intestinal tract. This homing and dispersal of activated T cells throughout the intestine is mediated by a variety of chemokines and adhesion molecules, among which the interaction of the integrin $\alpha 4 \beta 7$ on leukocytes and mucosal addressin cell adhesion molecule- 1 on postcapillary venules is particularly important.7,25,26 This mechanism allows for antigen-specific immune responses elicited in a single site to be rapidly disseminated throughout the intestinal tract. Thus, $\alpha 4 \beta 7+\mathrm{CD} 4+\mathrm{T}$ cells detected in the blood are believed to be recently activated, transitional or effector memory CD $4+\mathrm{T}$ cells, which are selectively homing to intestinal effector tissues. ${ }^{27}$ The data presented here indicate that a loss of this particular subset in blood correlates with the massive CD $4+\mathrm{T}$-cell depletion that occurs in the intestinal lamina propria in SIV, and presumably HIV-1 infection. This loss could either be the result of selective infection and destruction of these cells, or alternatively, may reflect the failure of the central memory pool of CD $4+\mathrm{T}$ cells to generate sufficient numbers of effector memory CD4 + T cells that can repopulate the intestine. Failure of this central memory pool has recently been hypothesized to have a major role in the development of immunosuppression and AIDS. ${ }^{28}$

Within days of infection, large numbers of memory CD4 + T cells are depleted in the intestinal lamina propria of HIV-infected humans, SIV-infected macaques, as well as non-progressing hosts such as sooty mangabeys and African green monkeys. 2,17,20,23,24 We and others have proposed that this rapid and selective depletion of intestinal memory CD $4+\mathrm{T}$ cells is mediated through direct viral infection and killing of these cells because of their high expression of CCR5 (permitting cells to be infected) and their state of activation, as HIV-1 selectively replicates in activated CD $4+$ T cells. ${ }^{8,15,16,18}$ In support of this, selective loss of activated memory CCR $5+\mathrm{CD} 4+\mathrm{T}$ cells can be detected in the intestine and other tissues in early SIV infection. ${ }^{8,15,16}$ However, memory CCR $5+$ CD $4+$ T cells are not limited to mucosal tissues. In early SIV infection, a selective loss of CCR $5+$ CD $4+$ $\mathrm{T}$ cells can clearly be detected in the peripheral blood. As SIV induces generalized immune activation, and because CCR5 + $\mathrm{T}$ cells are relatively rare in the peripheral blood, chronically infected animals may occasionally show similar or even higher levels of CD 4 + CCR $5+$ T cells in blood when compared with uninfected controls (unpublished observations). Furthermore, higher levels of CD4 + CCR5 + T cells have been reported in the blood of HIV-1 infected humans. ${ }^{29}$ Finally, in our cohort of animals controlling infection, CD4 + CCR $5+$ T cells seemingly returned to normal levels, similar to uninfected controls, despite persistently low levels of intestinal CD $4+\mathrm{T}$ cells, indicating that CCR5 expression in blood does not reflect the magnitude of intestinal CD4 + reconstitution. Thus, CCR5 levels in blood 

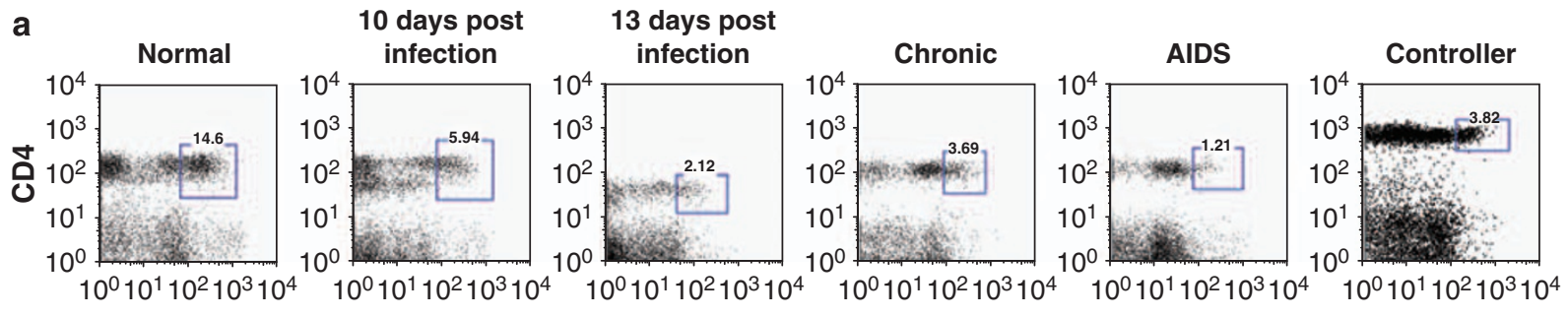

$\rightarrow \beta 7$ integrin

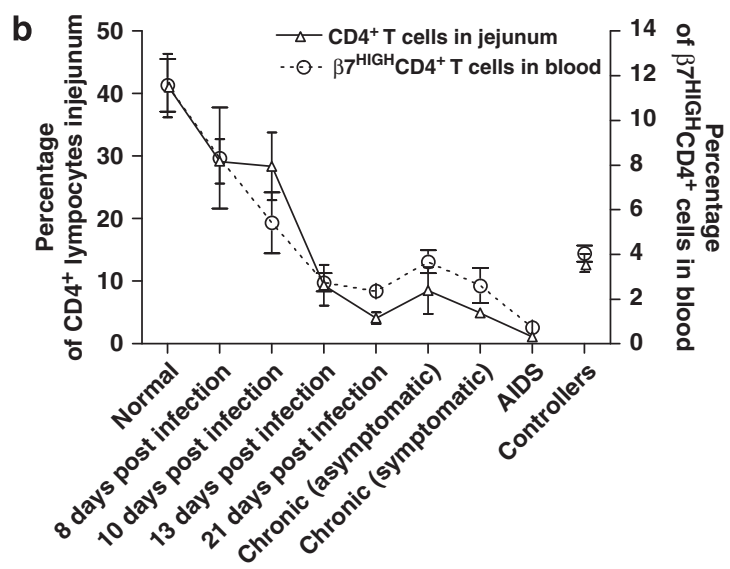

Figure 5 Loss of circulating $\beta 7^{\mathrm{HIGH}} \mathrm{CD} 4+\mathrm{T}$ cells in blood parallels the loss of intestinal CD4 + T cells throughout SIV infection. (a) Flow cytometry dot plots showing a rapid and persistent loss of $\beta 7^{\mathrm{HIGH}} \mathrm{CD} 4+\mathrm{T}$ cells in the blood. (b) Comparison of the loss of intestinal CD $4+T$ cells with the loss of $\beta 7{ }^{\mathrm{HIGH}} \mathrm{CD} 4+\mathrm{T}$ cells in the peripheral blood throughout infection. Note that the loss of $\beta 7{ }^{\mathrm{HIGH}} \mathrm{CD} 4+\mathrm{T}$ cells in blood directly parallels the loss of intestinal CD4 + T cells throughout SIV infection. Also note that the higher levels of intestinal CD4 + T cells in controllers is accompanied by higher levels of $\beta 7^{\mathrm{HIGH}} \mathrm{CD} 4+\mathrm{T}$ cells in the peripheral blood. Bars indicate s.e.m.

have not proven to be a reliable marker of systemic CD4 + T-cell depletion in chronic SIV or HIV-1 infection. Possible reasons for this include the lower frequency of CCR $5+\mathrm{T}$ cells in the circulation compared with $\alpha 4 \beta 7 \mathrm{CD} 4+\mathrm{T}$ cells, as well as the possibility CCR5 is dynamically upregulated in response to nonspecific inflammation in HIV infection, masking the effects of a selective depletion of these cells, at least in the blood.

Recently, Arthos et al. ${ }^{5}$ found that the HIV-1 envelope protein, gp120, binds to and signals by means of an activated form of integrin $\alpha 4 \beta 7$ on CD $4+T$ lymphocytes. These data suggest that $\alpha 4 \beta 7$ expression on $\mathrm{CD} 4+\mathrm{T}$ cells may have a more direct role in depletion of $\mathrm{CD} 4$ cells, and in the infection and persistence of SIV/HIV in intestinal tissues than previously recognized. The affinity of gp120 for $\alpha 4 \beta 7$ could have several roles in the selective depletion of $\mathrm{CD} 4+\mathrm{T}$ cells in intestinal or other tissues including; (i) using the $\alpha 4 \beta 7$ integrin for attachment and selective transport of the virus to intestinal tissues; (ii) facilitating direct infection of CD $4+$ CCR $5+T$ cells coexpressing $\alpha 4 \beta 7$ by approximating the virus to its major coreceptors; (iii) activating $\alpha 4 \beta 7^{\mathrm{HI}}$ cells potentially resulting in increased CCR5 expression, facilitating direct infection, and finally; (iv) facilitating infection of other cells by activation and formation of viral synapses, facilitating cell-to-cell spread of HIV- $1 .{ }^{5}$

These data show that the selectivity and degree of $\beta 7^{\mathrm{HIGH}} \mathrm{CD} 4+\mathrm{T}$-cell depletion in the blood during SIV infection parallels the loss of intestinal lamina propria CD4 $+\mathrm{T}$ cells.
Although the mechanisms behind this are not known, this may be due to increased activation (and thus more viral replication) of these cells because of selective binding of gp $120-\alpha 4 \beta 7$ on these cells. In vitro, gp 120/ $\alpha 4 \beta 7$ binding has been shown to increase expression of LFA-1, consistent with cell activation. ${ }^{5}$ Conceivably, such activation may also increase the expression of CCR 5 or other HIV-1 receptors, allowing cells to be more permissive to infection, or to support higher levels of viral replication. Although $\alpha 4 \beta 7+$ is not considered to be a receptor for viral entry into cells the loss of CD $4+\alpha 4 \beta 7+\mathrm{T}$ cells appears to be the result of killing of these cells, as there is clearly death and lysis of CD $4+\alpha 4 \beta 7+$ T cells (but not CD $3+C D 4$ neg cells) in the intestine in early SIV infection (Figure 4).

Finally, these data suggest a persistent and dramatic loss of $\beta 7^{\mathrm{HIGH}} \mathrm{CD} 4+\mathrm{T}$ cells in blood may correlate with progression to AIDS, and that restoration of $\beta 7^{\mathrm{HIGH}} \mathrm{CD} 4+\mathrm{T}$ cells may reflect the levels of partial reconstitution of intestinal CD4 $+\mathrm{T}$ cells. Thus, $\alpha 4 \beta 7$ may be critically involved in the pathogenesis of the intestinal CD4 + T-cell loss, and the development of AIDS in general, at least in SIV-infected macaques. Although responses in HIV-infected humans may differ, others have indeed shown a marked and persistent loss of $\alpha 4 \beta 7+\mathrm{CD} 4+\mathrm{T}$ cells in HIVinfected patients, even when on anti-retroviral therapy. ${ }^{30}$ Although these investigators did not examine intestinal CD4 + $T$ cells, these studies combined with others showing persistent loss of intestinal CD4 + T cells at all stages of HIV infection 

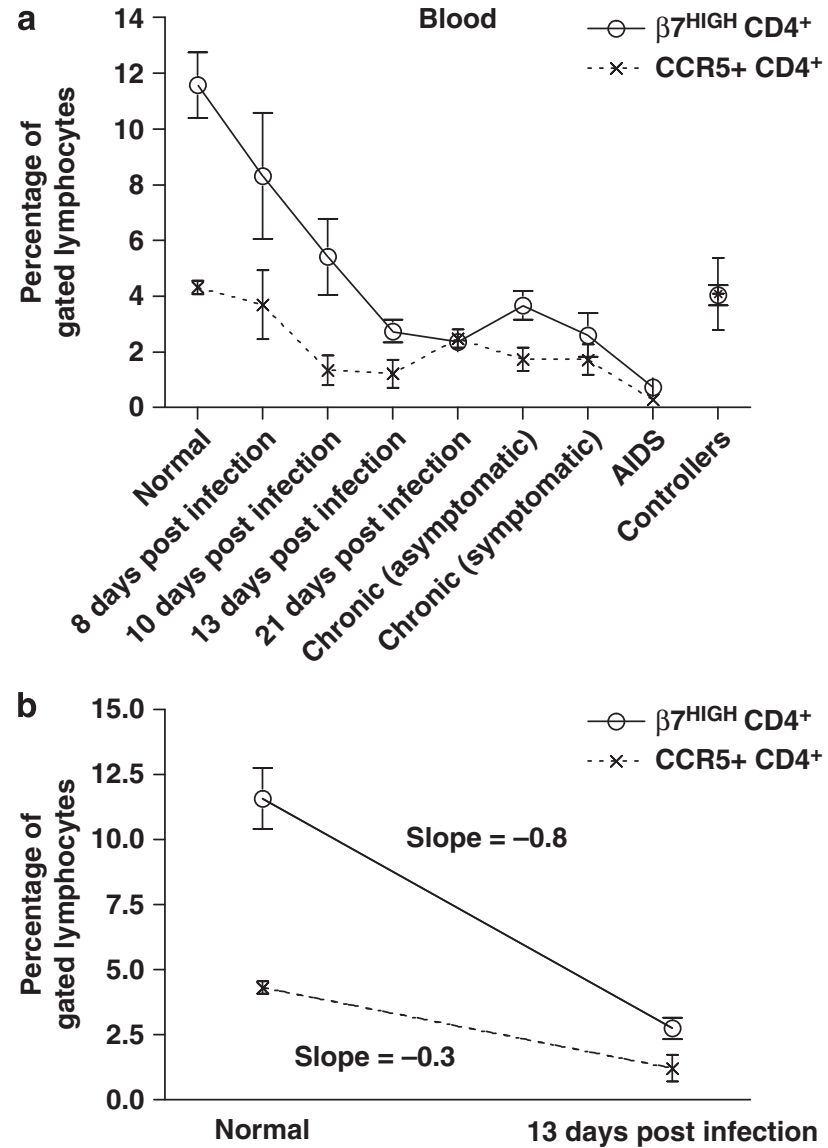

Figure 6 Comparison of changes in $\beta 7^{\mathrm{HIGH}} \mathrm{CD} 4+\mathrm{T}$ cells and CCR $5+$ CD $4+T$ cells from peripheral blood throughout SIV infection. (a) Comparison of peripheral blood $\beta 7{ }^{\mathrm{HIGH}} \mathrm{CD} 4+$, and CCR $5+\mathrm{CD} 4+$ T-cell subsets in normal and SIV-infected macaques. Note that there are significantly higher levels of $37^{\mathrm{HIGH}} \mathrm{CD} 4+\mathrm{T}$ cells than $\mathrm{CCR} 5+\mathrm{CD} 4+$ $T$ cells in the blood of normal macaques. Although there is a detectable loss of CCR $5+$ CD $4+T$ cells in the blood following SIV infection, it is neither as pronounced, nor as consistent in SIV-infected animals as the loss of $\beta 7{ }^{\mathrm{HIGH}} \mathrm{CD} 4+\mathrm{T}$ cells. Further, in animals controlling infection, $\mathrm{CCR} 5+\mathrm{CD} 4+\mathrm{T}$ cells are restored to levels similar to uninfected controls, whereas $\beta 7^{\mathrm{HIGH}} \mathrm{CD} 4+\mathrm{T}$ cells are only partially restored in the blood.

(b) Comparison of the slope of decline of $\beta 7^{\mathrm{HIGH}} \mathrm{CD} 4+\mathrm{T}$ cells to CCR5 + $T$ cells 13 days after SIV infection. Note that there is a more rapid and selective depletion of $\beta 7^{\mathrm{HIGH}} \mathrm{CD} 4+\mathrm{T}$ cells in the blood as compared with CCR5 + CD4 + T cells in SIV infection. SIV, simian immunodeficiency virus.

suggest that tracking $\beta 7$ expression in blood may be a useful surrogate marker for monitoring intestinal CD4 + T-cell depletion and reconstitution in HIV-1 vaccine and therapeutic studies. Clearly, more studies are needed to validate these findings in HIV-infected patients, but these results suggest that $\alpha 4 \beta 7$ expression may have a major role in the pathogenesis of HIV infection and the development of AIDS.

\section{MATERIALS AND METHODS}

\section{Animals and virus}

A total of 46 adult rhesus macaques (Macaca mulatta) of Indian origin on SIV pathogenesis studies were examined in this study. All animals were housed at the Tulane National Primate Research Center in accordance with the standards of
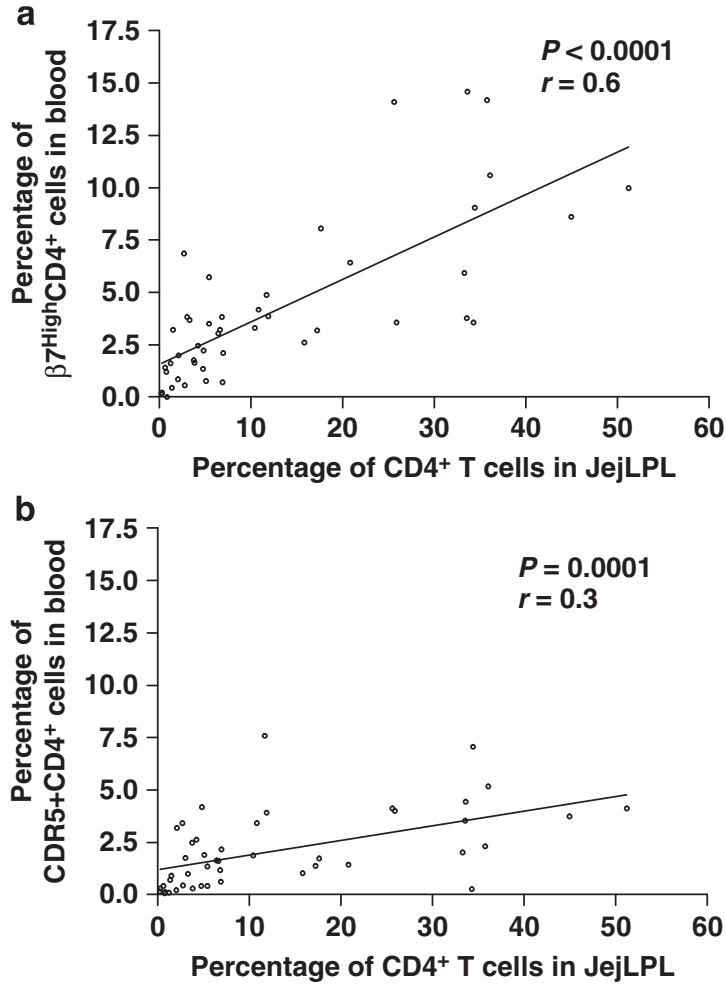

Figure 7 Linear regression analysis comparing the percentage of intestinal CD $4+\mathrm{T}$ cells peripheral blood $\beta 7^{\mathrm{HIGH}} \mathrm{CD} 4+\mathrm{T}$ cells $(\mathbf{a})$, and CCR5 +CD4 + T cells (b) in normal and SIV-infected macaques. Although a positive correlation is observed in both, note there is a much stronger positive correlation between circulating $37^{\mathrm{HIGH}} \mathrm{CD} 4+$

$T$ cells and intestinal CD4 + T cells than for circulating CCR $5+C D 4+$ $T$ cells. Correlation coefficients were determined using the Spearman's coefficient of correlation and linear regression was used to obtain $r^{2}$ values. SIV, simian immunodeficiency virus.

the Association for Assessment and Accreditation of Laboratory Animal Care International standards. All studies were reviewed and approved by the Tulane University Institutional Animal Care and Use Committee. Of these, five uninfected animals were killed for tissue collection as controls, and another 41 were infected with either SIV or SHIV as follows; Thirty-seven macaques were infected with SIV either intravenously $(n=27)$, intrarectally $(n=6)$ or intravaginally $(n=4)$ with either wild type SIVmac251 $(n=31)$, SIVdeltaB670 $(n=2)$ (viruses provided by Preston Marx) or molecularly cloned SIVmac239 $(n=4)$ (virus courtesy of Ronald Desrosiers). These 37 SIV-infected macaques were humanely killed for tissue collection at the time points shown in Figure 3, including early ( 8 days $(n=4), 10$ days $(n=3)$, 13 days $(n=3)$ and 21 days $(n=4))$ post infection, chronic infection with either no overt signs of disease (chronic asymptomatic, $n=8$ ) or with illness that could not be definitively attributed to AIDS (e.g., nonresponsive diarrhea, weight loss, etc; $n=6$ ), and nine animals with overt signs of AIDS. All animals killed early in infection (21 days or less) were intravenously infected with SIVmac251 to reduce variation that can occur with mucosal inoculations, but we grouped chronically infected animals irrespective of inoculum or route because we have found that the pathogenesis of chronic infection is similar with these viruses, 
regardless of the route of inoculation. SIV-infected macaques killed with AIDS ( $n=9$ ), all had AIDS-defining lesions and/or opportunistic infections including Pneumocystis carinii pneumonia $(n=6)$; disseminated Mycobacterium avium infection $(n=2)$ or SIV encephalitis $(n=1)$. The remaining four animals ("controllers") refers to four macaques infected with either SIVmac251 $(n=1)$, SHIV89.6P $(n=2)$ or SHIV-KU $(n=1)$ which became infected and originally had high-peak plasma viremia, but subsequently controlled viral replication to undetectable levels ( $<125$ copies per $\mathrm{ml}$ ) in plasma. Intestinal samples from these animals were collected by endoscopic biopsy, as they remain clinically healthy.

\section{Cell isolation and flow cytometry}

Tissues were collected from the jejunum, ileum, colon, spleen, mesenteric, and axillary lymph nodes, and thymus within minutes of necropsy and transported to the lab on ice for immediate processing. Lymphocytes from the intestine and other tissues were isolated and stained for flow cytometry as previously described. ${ }^{15}$ Peripheral blood, spleen, and bone marrow cells were stained using a whole blood lysis technique as previously described.$^{15}$ Blood and intestinal lymphocytes from all 46 animals were examined by four color flow cytometry with fluorescently conjugated monoclonal antibodies to CD4-APC, CD8-PerCP, CD45RA-FITC, or CD3-FITC combined with CCR5-PE (clone $3 A 9)$, or $\beta 7$ integrin-PE (BD Biosciences, San Diego, CA) in separate tubes. Samples were acquired on a FACS Calibur flow cytometer (Becton Dickinson, Franklin Lakes, NJ) and analyzed with Flowjo software (Tree star, Ashland, OR). To further characterize $\beta 7^{\mathrm{HIGH}} \mathrm{CD} 4+$ cells in blood, an additional five normal macaques were examined by nine-color flow cytometry using appropriately diluted, directly conjugated monoclonal antibodies to CD95-FITC or CD45RA-FITC, $\beta 7$ integrin-PE, CD49d/integrin $\alpha 4$-PE-Cy5, HLADR-PE-Cy7, CD28-APC, CD69-APC-Cy7, CD3-Pac. Blue (BD Biosciences) CD8- PE-TR (Caltag Laboratroies, Carlsbad, $\mathrm{CA}$ ), and CD4-Qdot655 (NIH), followed by red blood cell lysis and wash with dPBS/BSA. These samples were resuspended with BD Stabilizing Fixative (BD Biosciences) and acquired on LSRII flow cytometer (Becton Dickinson). Data were analyzed with Flowjo software (Tree star).

\section{Immunohistochemistry}

Three color immunofluorescent staining for $\beta 7, \mathrm{CD} 4$, and CD3 ( $\mathrm{T}$ cells) was performed on selected animals (uninfected and through day 21 infection) to visualize and phenotype the distribution of $\beta 7+\mathrm{T}$-cell subsets in tissues by confocal microscopy as previously described. ${ }^{31}$ In brief, tissues were stained using unconjugated primary antibodies and then with secondary antibodies conjugated to either Alexa 488 (green), Alexa 568 (red) or Alexa 633 (blue)(Molecular Probes, Eugene, OR). Confocal microscopy was performed using a Leica TCS SP2 confocal microscope equipped with three lasers (Leica Microsystems, Exton, PA). Individual optical slices represent $0.2 \mathrm{~mm}$, and 32-62 optical slices were collected at $512 \times 512$ Q1 pixel resolution. NIH Image (version 1.62) and Adobe Photoshop (version 7.0) were used to assign colors to the channels collected.

\section{Statistics}

Graphical presentation and statistical analysis of the data were performed using GraphPad Prism 4.0 (GraphPad Software, SanDiego, CA). Comparisons between three or more groups were analyzed by a one-way ANOVA and Dunnett's Multiple Comparison Test for significant differences $(P<0.05)$. The Dunnett's test was also used when comparing controls (one group) to other groups. Correlations between samples were calculated and expressed using the Spearman's coefficient of correlation. The rate of cell loss was determined using Linear Regression analysis.

\section{ACKNOWLEDGMENTS}

We thank Linda Green, Janell LeBlanc, Maryjane Dodd, Kelsi Rasmussen, Robin Rodriguez, and Maury Duplantis for their technical assistance, and Julie Bruhn, Calvin Lanclos, and Desiree Waguespacek for their expertise in flow cytometry. We thank Jason Dufour and Morgan Singletary for their veterinary expertise and the animal care staff for animal care. The work was supported in part by NIH Grants RR00164, AI049080, AA013563, and AI062410. The authors have no conflicting or competing financial interests.

\section{DISCLOSURE}

The authors declared no conflict of interest.

(c) 2009 Society for Mucosal Immunology

\section{REFERENCES}

1. Mehandru, S. et al. Lack of mucosal immune reconstitution during prolonged treatment of acute and early HIV-1 infection. PLOS Med 3, e484 (2006).

2. Guadalupe, M. et al. Severe CD4+ T-cell depletion in gut lymphoid tissue during primary human immunodeficiency virus type 1 infection and substantial delay in restoration following highly active antiretroviral therapy. J Virol 77, 11708-11717 (2003).

3. Guadalupe, M. et al. Viral suppression and immune restoration in the gastrointestinal mucosa of human immunodeficiency virus type 1-infected patients initiating therapy during primary or chronic infection. J Viro/ 80, 8236-8247 (2006)

4. Veazey, R.S. \& Lackner, A.A. Impact of antiretroviral therapy on intestinal lymphoid tissues in HIV infection. PLoS Med 3, e515 (2006).

5. Arthos, J. et al. HIV-1 envelope protein binds to and signals through integrin alpha4beta7, the gut mucosal homing receptor for peripheral T cells. Nat Immuno/ 9, 301-309 (2008).

6. Berlin, C. et al. a4 integrins mediate lymphocyte attachment and rolling under physiologic flow. Cell 80, 413-422 (1995).

7. Butcher, E.C. \& Picker, L.J. Lymphocyte homing and homeostasis. Science 272, 60-66 (1996).

8. Veazey, R.S. et al. Dynamics of CCR5 expression by CD4(+) T cells in lymphoid tissues during simian immunodeficiency virus infection. J Virol 74, 11001-11007 (2000).

9. Poles, M.A., Elliott, J., Taing, P., Anton, P.A. \& Chen, I.S. A preponderance of CCR5(+) CXCR4(+) mononuclear cells enhances gastrointestinal mucosal susceptibility to human immunodeficiency virus type 1 infection. J Virol 75, 8390-8399 (2001).

10. Lackner, A.A. \& Veazey, R.S. Current Concepts in AIDS Pathogenesis: insights from the SIV/Macaque Model. Annu Rev Med 58, 461-476 (2007).

11. Andrew, D.P., Rott, L.S., Kilshaw, P.J. \& Butcher, E.C. Distribution of alpha 4 beta 7 and alpha $E$ beta 7 integrins on thymocytes, intestinal epithelial Iymphocytes and peripheral lymphocytes. Eur J Immunol 26, 897-905 (1996).

12. Rodriguez, M.W., Paquet, A.C., Yang, Y.H. \& Erle, D.J. Differential gene expression by integrin beta $7+$ and beta 7 - memory T helper cells. BMC Immunol 5, 13 (2004).

13. Ericsson, A., Svensson, M., Arya, A. \& Agace, W.W. CCL25/CCR9 promotes the induction and function of CD103 on intestinal intraepithelial lymphocytes. Eur J Immuno/ 34, 2720-2729 (2004). 


\section{ARTICLES}

14. Mora, J.R. \& von Andrian, U.H. T-cell homing specificity and plasticity: new concepts and future challenges. Trends Immuno/ 27, 235-243 (2006).

15. Veazey, R.S. et al. Identifying the target cell in primary simian immunodeficiency virus (SIV) infection: highly activated memory CD4(+) T cells are rapidly eliminated in early SIV infection in vivo. J. Viro/ 74, 57-64 (2000).

16. Mattapallil, J.J., Douek, D.C., Hill, B., Nishimura, Y., Martin, M. \& Roederer, M. Massive infection and loss of memory CD4+T cells in multiple tissues during acute SIV infection. Nature 434, 1093-1097 (2005).

17. Brenchley, J.M. et al. CD4+ T cell depletion during all stages of HIV disease occurs predominantly in the gastrointestinal tract. J Exp Med 200, 749-759 (2004).

18. Schnittman, S.M., Lane, H.C., Greenhouse, J., Justement, J.S., Baseler, M. \& Fauci, A.S. Preferential infection of CD4+ memory cells by human immunodeficiency virus type 1: evidence for a role in the selective T-cell functional defects observed in infected individuals. Proc. Natl. Acad. Sci 87, 6058-6062 (1990).

19. Brenchley, J.M. et al. Microbial translocation is a cause of systemic immune activation in chronic HIV infection. Nat Med 12, 1365-1371 (2006).

20. Veazey, R.S., Marx, P.A. \& Lackner, A.A. The mucosal immune system: primary target for HIV infection and AIDS. Trends Immunol 22, 626-633 (2001).

21. Picker, L.J. et al. Immunopathogenesis of acute AIDS virus infection. Curr Opin Immunol 18, 399-405 (2006).

22. Mackay, C.R. \& Sallusto, F. A new role for CCR5 in innate immunity--binding to bacterial heat shock protein 70. Eur J Immunol 36, 2293-2295 (2006).
23. Veazey, R.S. et al. Gastrointestinal tract as a major site of CD4+ T-cell depletion and viral replication in SIV infection. Science 280, 427-431 (1998).

24. Mehandru, S. et al. Primary HIV-1 infection is associated with preferential depletion of CD4+ T lymphocytes from effector sites in the gastrointestinal tract. J Exp Med 200, 761-770 (2004)

25. Berlin, C. et al. Alpha 4 beta 7 integrin mediates lymphocyte binding to the mucosal vascular addressin MAdCAM-1. Cell 74, 185-195 (1993).

26. Briskin, M. et al. Human mucosal addressin cell adhesion molecule-1 is preferentially expressed in intestinal tract and associated lymphoid tissue. Am J Pathol 151, 97-110 (1997).

27. Campbell, D.J. \& Butcher, E.C. Rapid acquisition of tissue-specific homing phenotypes by CD4(+) T cells activated in cutaneous or mucosal lymphoid tissues. J Exp Med 195, 135-141 (2002).

28. Okoye, A. et al. Progressive CD4+ central memory T cell decline results in CD4+ effector memory insufficiency and overt disease in chronic SIV infection. J Exp Med 204, 2171-2185 (2007).

29. Ostrowski, M.A. et al. Expression of chemokine receptors CXCR4 and CCR5 in HIV-1-infected and uninfected individuals. J Immunol 161, 3195-3201 (1998).

30. Krzysiek, R. \& AOPAS Group Preferential and persistent depletion of CCR5+ T-helper lymphocytes with nonlymphoid homing potential despite early treatment of primary HIV infection. Blood 98, 3169-3171 (2001).

31. Borda, J.T. et al. Cell tropism of simian immunodeficiency virus in culture is not predictive of in vivo tropism or pathogenesis. Am J Pathol 165 2111-2122 (2004) 